\title{
Improvement of sulphur mustard-induced chronic pruritus, quality of life and antioxidant status by curcumin: results of a randomised, double-blind, placebo-controlled trial
}

\author{
Yunes Panahi ${ }^{1}$, Amirhossein Sahebkar ${ }^{1,2 *}$, Mojtaba Amiri ${ }^{1}$, Seyyed Masoud Davoudi ${ }^{1}$, \\ Fatemeh Beiraghdar $^{3}$, Seyyedeh Leila Hoseininejad ${ }^{1}$ and Marjan Kolivand ${ }^{1}$ \\ ${ }^{1}$ Chemical Injuries Research Center, Baqiyatallab University of Medical Sciences, Tehran, Iran \\ ${ }^{2}$ Biotechnology Research Center, School of Pharmacy, Mashbad University of Medical Sciences, Mashbad, Iran \\ ${ }^{3}$ Nephrology and Urology Research Center, Baqiyatallah University of Medical Sciences, Tehran, Iran
}

(Submitted 15 April 2011 - Final revision received 24 October 2011 - Accepted 29 October 2011 - First published online 18 November 2011)

\begin{abstract}
Skin is among the first and most heavily damaged organs upon sulphur mustard (SM) exposure. Pruritus is the most common chronic skin complication of SM, which adversely affects the quality of life (QoL). However, current therapies for the management of SM-induced pruritus are very limited and associated with side effects. The present trial investigated the efficacy of curcumin in the alleviation of SM-induced chronic pruritic symptoms. A total of ninety-six male Iranian veterans (age 37-59 years) were randomised to receive either curcumin $(1 \mathrm{~g} / \mathrm{d}, n$ 46) or placebo $(n 50)$ for 4 weeks. Serum concentrations of substance P and activities of antioxidant enzymes were measured at baseline and at the end of the trial. Assessment of pruritus severity was performed using the pruritus score, visual analogue scale (VAS) and scoring atopic dermatitis (SCORAD) index. QoL was evaluated using the Dermatology Life Quality Index (DLQI) questionnaire. Serum concentrations of substance $\mathrm{P}(P<0.001)$ as well as activities of superoxide dismutase $(P=0.02)$, glutathione peroxidase $(P=0.006)$ and catalase $(P<0.001)$ were significantly reduced in the curcumin group, while no significant change was observed in the placebo group. Curcumin supplementation was also associated with significant reductions in measures of pruritus severity including the pruritus score $(P<0 \cdot 001)$, VAS score $(P<0 \cdot 001)$, overall $(P<0.001)$ and objective SCORAD $(P=0 \cdot 009)$, and DLQI's first question $(P<0 \cdot 001)$. None of these measures was significantly changed in the placebo group. As for the QoL, although DLQI scores decreased in both groups $(P<0.001$ and $P=0.003$ in the curcumin and placebo groups, respectively), the magnitude of reduction was significantly greater in the curcumin group $(P<0 \cdot 001)$. In conclusion, curcumin may be regarded as a natural, safe, widely available and inexpensive treatment for the management of SM-induced chronic pruritus.
\end{abstract}

Key words: Curcumin: Pruritus: Sulphur mustard: Antioxidants: Quality of life

Bis(2-chloroethyl)sulphide, commonly known as sulphur mustard (SM), is a compound with potent alkylating, vesicant, blister-forming, cytotoxic, mutagenic and carcinogenic properties $^{(1)}$. SM has been the most frequently used chemical warfare agent during the past century. Following its first use in World War I, SM has been deployed in several other military combats. Unfortunately, this agent was extensively used against Iranian army and civilians during the Iraq-Iran war (1980-8), leading to about 100000 chemically injured victims, of whom a considerable fraction still suffer from late SM complications ${ }^{(2-4)}$. Skin is one of the most vulnerable organs upon SM exposure, and this is due to its high surface area ${ }^{(5)}$. Cutaneous complications of SM could be classified into acute and chronic. One of the most prevalent chronic skin complications of $\mathrm{SM}$ is pruritus ${ }^{(6)}$. In addition to its high prevalence, pruritus has been reported to impair the patient's quality of life $(\mathrm{QoL})^{(7-9)}$. Therefore, its management is of high priority in patients with SMinduced pruritic skin lesions.

Although the exact pathophysiology of SM-induced dermatotoxicity is not yet fully elucidated, a number of pathomechanisms have been proposed. One of these mechanisms is based on the development of oxidative stress in intoxicated patients. There has been a considerable body of evidence on the pivotal role of oxidative stress in the pathogenesis of both acute and chronic complications of $\mathrm{SM}^{(10,11)}$.

Abbreviations: DLQI, Dermatology Life Quality Index; GPx, glutathione peroxidase; QoL, quality of life; SCORAD, scoring atopic dermatitis; SM, sulphur mustard; SOD, superoxide dismutase; VAS, visual analogue scale. 
Pruritus is the most common complaint of SM-exposed patients who suffer from chronic skin lesions. Being present in about $70-90 \%$ of patients, this annoying complication has been reported to significantly affect several aspects of daily functioning and cause sleeping, psychological and sexual disorders, thereby leading to a decreased $\mathrm{QOL}^{(7-9)}$. During the past decades, several mediators have been identified for pruritus. One of the most important mediators is substance $\mathrm{P}$, which is a neuropeptide with a key role in pain perception and inflammation. As pruritus is regarded as a nociceptive stimulus, this neurotransmitter is involved in the sensation of pruritus. Intradermal injection of substance $\mathrm{P}$ has been shown to induce pruritus in both animals and human subjects. This effect is mainly due to the neuronal sensitisation and activation of mast cells through the interaction of substance $\mathrm{P}$ with neurokinin receptors, resulting in histamine release $^{(12,13)}$. Aside from mast cell activation, substance $\mathrm{P}$ has direct pro-inflammatory properties and induces the expression and release of inflammatory cytokines such as IL-1 and TNF- $\alpha^{(14,15)}$.

In spite of its importance, few studies have addressed therapeutic anti-pruritic approaches in SM-exposed subjects ${ }^{(16-20)}$. Treatment options currently employed for the control of SM-induced chronic pruritus are mainly symptomatic and generally limited to antihistamines, local moisturisers and, in particular, topical corticosteroids. Since long-term application of topical corticosteroids is associated with several side effects such as skin atrophy, striae, telangiectasia, acne, etc. ${ }^{(21)}$, there is an apparent demand for the introduction of newer and safer therapeutic agents that could effectively alleviate pruritic symptoms.

Curcumin (diferuloylmethane; Fig. 1) is the yellow bioactive ingredient of turmeric (powdered rhizomes of Curcuma longa L.) that has been used as a dietary spice and versatile traditional medicine for thousands of years, in particular in Indian Ayurvedic medicine. Over the past decades, this polyphenolic compound has been successfully applied against a wide variety of disorders, leading to the identification of numerous promising therapeutic benefits such as anticancer, anti-inflammatory, antioxidant, immunomodulatory, cardio- and neuroprotective activities ${ }^{(22-24)}$. Regarding the role of oxidative stress and inflammation in the pathogenesis of SM-induced chronic skin complications ${ }^{(10,25)}$, curcumin could represent a potential medication for the alleviation of symptoms. Therefore, the present trial sets out to investigate the therapeutic efficacy of supplementation with curcumin in the attenuation of SM-induced pruritus.<smiles>COc1cc(/C=C/C(=O)CC(=O)/C=C/c2ccc(O)c(Cl)c2)ccc1O</smiles>

Fig. 1. Chemical structure of curcumin.

\section{Methods}

\section{Participants}

The present study was a randomised, double-blind, doublecentre and placebo-controlled clinical trial, performed in the Veterans Clinic of Tehran and Ilam (west of Iran). Included subjects were male Iranian veterans of the Iraq-Iran war (age range 37-59 years), who were suffering from chronic pruritic skin lesions. These patients were exposed to mustard gas about 20 years ago and had been treated by skin decontamination and/or antibiotics in the first stages following SM exposure. The exposure was confirmed by documented development of blisters in the exposed areas of the skin and transient visual deterioration lasting for several days and associated respiratory symptoms. Patients were excluded if itching resulted from systemic or cutaneous non-chemical diseases, or if they had received any topical treatments within 1 month before the study.

Included patients ( $n$ 96) were randomised to receive curcumin ( $1 \mathrm{~g} / \mathrm{d}$; curcumin group, $n$ 46) or matched placebo (placebo group, $n$ 50) for a period of 4 weeks. The groups were matched regarding age (47.5 (SD 10.7) and 48.3 (SD 8.5) years in the curcumin and placebo groups, respectively). Curcumin was administered in the form of C3 Complex ${ }^{\circledR}$ capsules (Sami Labs Limited) containing $500 \mathrm{mg}$ curcuminoids plus $5 \mathrm{mg}$ Bioperine ${ }^{\circledR}$. Bioperine ${ }^{\circledR}$ is an extract obtained from black pepper (Piper nigrum L.) or long pepper (Piper longum L.), and contains $95 \%$ piperine which is a well-documented bioavailability enhancer ${ }^{(26)}$. Placebo capsules used in the study were shape- and size-matched, and contained piperine $(5 \mathrm{mg})$.

Patients were visited by a board-certified dermatologist at baseline and at the end of the treatment duration. The study was conducted according to the guidelines laid down in the Declaration of Helsinki ${ }^{(27)}$. The study protocol was approved by the Ethics Committee of the Baqiyatallah University of Medical Sciences, and written informed consent was obtained from the participants.

\section{Assessment of pruritus severity}

Assessment of pruritus severity was performed using the visual analogue scale (VAS), pruritus score and scoring atopic dermatitis (SCORAD) index. The VAS was designed as a $100 \mathrm{~mm}$ horizontal line without scaling, in which 0 was marked as 'no pruritus' and 100 was marked as 'unbearable pruritus'. Patients were then instructed to place a vertical mark reflecting their pruritus severity. Based on the VAS score, the severity of disease could be classified into mild (0-3), moderate (4-6) or severe ${ }^{(7-10)}$.

The pruritus score was calculated according to the questionnaire before and after the treatment course for each patient ${ }^{(28)}$. In the questionnaire, the entire $24 \mathrm{~h}$ period was divided into three periods: morning, the time from getting up until noon; afternoon, the time from noon until going to bed; and night. We allotted 1 point for the report of pruritus in each period, and a maximum of 3 points for patients who complained of pruritus during all the three periods. The scores of severity, 
distribution and frequency of pruritus were recorded separately for the morning and the afternoon as follows:

Severity: itching without the need to scratch (1 point); itching with an occasional need to scratch (2 points); frequent scratching (3 points); no itching relief with scratching (4 points); itching with discomfort all the time ( 5 points).

Distribution: for each body part - arms, trunk, legs, face and head ( 1 point), with a maximum of 5 points; generalised itching ( 5 points).

Frequency: itching in two periods of less that $10 \mathrm{~min}$ or one period of more than $10 \mathrm{~min}$ ( 1 point); itching in ten periods of less than $10 \mathrm{~min}$ or five periods of more than 10 min (5 points).

Sleep duration and waking up were recorded at night as follows:

Sleep duration: absence of sleep (10 points); $7 \mathrm{~h}$ or more of night sleep (0 points). Other points were scored by deducting the number of sleeping hours from 10 .

Waking up: for each awakening due to pruritus (1 point); a maximum of 5 points for five or more episodes.

The overall summed pruritus score ranges from 1 to 48, with a higher score indicating more severe pruritus. This range is divided into three equal sections as mild (1-16 points), moderate (17-32 points) and severe (33-48 points).

The SCORAD index was also applied to assess the extent and intensity of cutaneous complications. This index was developed by the European Task Force on Atopic Dermatitis in 1993 based on a broad consensus by dermatologists ${ }^{(29)}$. This index consists of three parts: (A) extent of the disorder (as a percentage of the whole body), which is calculated based on the rule of nines (head and neck (9\%), upper limbs ( $9 \%$ each), lower limbs (18\% each), anterior trunk (18\%), back (18\%) and $1 \%$ each for genitals, each palm and the back of each hand); (B) intensity of the disorder, which comprises assessment and grading of six items - erythema, oedema/papulation, excoriations, lichenification, oozing/ crusts and dryness; and (C) subjective symptoms including pruritus and sleeplessness. Based on the mathematical model of main component analysis in a previous landmark trial, the following formula has been suggested for the calculation of the total SCORAD score: $\mathrm{A} / 5+7 \mathrm{~B} / 2+\mathrm{C}^{(29)}$. The maximum scores of parts A, B and C are 100, 18 and 20, respectively. Therefore, the SCORAD index would range between 0 and 103. The objective SCORAD is a modified form of the SCORAD index, in which the subjective part that appeared to be a source of large variations - has been eliminated and the concentration is focused on objective signs (extent and intensity). The range of the objective SCORAD lies between 0 and 83 . Based on the overall SCORAD and the objective SCORAD, the severity of disease could be classified into mild $(<15$ for the objective SCORAD and $<25$ for the overall SCORAD), moderate (15-40 for the objective SCORAD and 25-50 for the overall SCORAD) or severe ( $\geq 40$ for the objective SCORAD and $\geq 50$ for the overall SCORAD).

\section{Assessment of quality of life}

QoL was evaluated using the Dermatology Life Quality Index (DLQI). The DLQI is a simple, practical and widely used index for the assessment of health-related QoL and has previously been employed in an Iranian population including subjects with SM-induced chronic skin lesions ${ }^{(7)}$. The DLQI questionnaire consists of ten questions under six headings: symptoms and feelings (questions 1 and 2), daily activities (questions 3 and 4), leisure (questions 5 and 6), work and school (question 7), personal relationships (questions 8 and 9) and treatment (question 10). Each question has a maximum score of 3 and options of 'very much' (score 3), 'a lot' (score 2), 'a little' (score 1) and 'not at all' (score 0). In addition, a '0' score is allocated to 'not relevant' response and unanswered questions. The DLQI total score is calculated by summing the scores of all questions, resulting in a score range of $0-30$, in which higher scores are associated with more severe impairments of QoL. As the first question of the DLQI deals with pruritus severity over the previous week, it could be separately analysed as a pruritus severity index. In the present study, the complete DLQI, the DLQI without the first question (DLQI $-\mathrm{Q}_{1}$ ) and the first question of the DLQI were individually analysed.

\section{Biochemical analyses}

Blood samples were collected at baseline and at the end of the trial. Serum substance $\mathrm{P}$ concentrations, together with the activities of superoxide dismutase (SOD), glutathione peroxidase (GPx) and catalase, were measured using commercial kits.

\section{Statistical analyses}

Statistical analyses were performed using SPSS software for Windows (version 11.5; SPSS, Inc.). Data were expressed as means and standard deviations. Data were assessed for normality using the Kolmogorov-Smirnov test. Group comparisons were made using the independent-samples $t$ test and paired-samples $t$ test for normally distributed data, or the Mann-Whitney $U$ test and Wilcoxon signed-rank test for non-normally distributed data. Categorical variables were compared using the $\chi^{2}$ or Fisher's exact test. Bivariate correlations between changes in all assessed efficacy (pruritus score, VAS score, overall SCORAD and DLQI) and biochemical (serum substance $\mathrm{P}, \mathrm{SOD}, \mathrm{GPx}$ and catalase) measures were evaluated in the curcumin and placebo groups using Pearson's (for normally distributed data) or Spearman's (for non-normally distributed data) rank correlation coefficients. Stepwise multiple linear regression analysis was used to identify the independent parameters that are related to the evaluated efficacy measures. Changes (pre-trial $v$. post-trial) in pruritus score, VAS score, overall SCORAD and DLQI were entered into the model as the dependent variable. Predictor variables included changes in serum substance $\mathrm{P}$ concentration, SOD, GPx and catalase activity. A two-tailed $P$ value of $<0.05$ was considered to be statistically significant. 


\section{Results}

In the present study, a total of ninety-six patients entered into the study, of whom eighty completed the study (forty per group) and were included in the final analyses. Of the sixteen patients who did not complete the study, six discontinued the study treatment in the curcumin group (three because of gastrointestinal side effects, one because of fear of side effects, one because of inability to return to the clinic because of long distance and one due to unknown reason) and ten in the placebo group (seven because of no treatment response or worsening of symptoms, two because of gastrointestinal side effects and one because of inability to return to the clinic because of long distance). There was no significant difference in the dropout rate between the groups.

\section{Effect of curcumin supplementation on serum substance $P$,} superoxide dismutase, glutathione peroxidase and catalase activity

Baseline values for serum substance P, SOD and catalase were comparable between the curcumin and placebo groups. In contrast, baseline GPx activity was significantly higher in the curcumin group compared with the placebo group $(P=0 \cdot 004 ;$ Table 1$)$. Supplementation with curcumin was associated with a marked decrease in serum substance $\mathrm{P}$ concentrations ( $-29 \%, P<0.001)$, while there were significant elevations in serum SOD (21\%, $P=0.020)$, GPx $(20 \%$, $P=0.006)$ and catalase $(30 \%, P<0.001)$ activities (Table 1$)$. There was no significant change in any of the aforementioned parameters in the placebo group.

\section{Effect of curcumin supplementation on the pruritus, visual} analogue scale and scoring atopic dermatitis scores

Pruritus scores were comparable between the curcumin and placebo groups at baseline, whereas baseline VAS $(P=0.003)$, overall SCORAD $(P=0.001)$ and objective
SCORAD $(P=0.009)$ scores were higher in the curcumin group compared with the placebo group. Supplementation with curcumin was associated with a marked decline in pruritus $(-30 \%)$, VAS $(-48 \%)$, overall SCORAD $(-58 \%)$ and objective SCORAD ( $-58 \%)$ scores $(P<0 \cdot 001$; Fig. 2). In contrast, no significant difference in these parameters was observed in the placebo group $(P>0.05$; Fig. 2$)$.

\section{Effect of curcumin supplementation on quality of life and the rate of pruritus improvement}

Baseline DLQI scores were not significantly different between the groups. The DLQI was significantly decreased by the end of the trial in both curcumin $(-30 \%, P<0.001)$ and placebo ( $-6 \%, P=0.003$ ) groups (Fig. 2). DLQI $-\mathrm{Q}_{1}$ scores were significantly decreased in both curcumin $(-24 \%, P<0.001)$ and placebo $(-6 \%, P=0.005)$ groups (Fig. 2). The $\mathrm{Q}_{1}$ score was significantly decreased in the curcumin group $(-75 \%$, $P<0.001)$, while there was no significant change in the placebo group (Fig. 2). The rates of improvement for all four efficacy measures were clearly higher in the curcumin group compared with the placebo group $(P<0 \cdot 001$; Table 2$)$.

\section{Bivariate analysis}

In the curcumin group, the only significant correlation was between changes in pruritus score and serum SOD activity $(r-0.32, P=0 \cdot 041)$. In the placebo group, a significant correlation was only observed between changes in pruritus score and VAS score $(r 0 \cdot 40, P=0 \cdot 010)$. In addition, a borderline significant correlation was found in the placebo group between changes in serum SOD and GPx activities ( $r 0 \cdot 31, P=0 \cdot 051)$.

\section{Multiple regression analysis}

In the curcumin group, change in serum SOD activity was found as a significant predictor of pruritus score variations

Table 1. Effect of curcumin v. placebo on the evaluated biochemical parameters

(Mean values and standard deviations)

\begin{tabular}{|c|c|c|c|c|c|c|c|c|c|c|}
\hline & \multicolumn{5}{|c|}{ Curcumin } & \multicolumn{5}{|c|}{ Placebo } \\
\hline & \multicolumn{2}{|c|}{ Pre-trial } & \multicolumn{2}{|c|}{ Post-trial } & \multirow[b]{2}{*}{$P$} & \multicolumn{2}{|c|}{ Pre-trial } & \multicolumn{2}{|c|}{ Post-trial } & \multirow[b]{2}{*}{$P$} \\
\hline & Mean & SD & Mean & SD & & Mean & SD & Mean & SD & \\
\hline Substance P (pg/ml) & 3.35 & 0.67 & $2 \cdot 34$ & 0.72 & $<0.001$ & $3 \cdot 34$ & 0.55 & $3 \cdot 18$ & 0.44 & $>0.05$ \\
\hline $\operatorname{SOD}(\mu \mathrm{Kat} / \mathrm{l})$ & 57.68 & 12.67 & 65.85 & 13.84 & 0.020 & 58.01 & $12 \cdot 17$ & $57 \cdot 01$ & 8.84 & $>0.05$ \\
\hline GPx $(\mu \mathrm{Kat} / \mathrm{l})$ & 63.68 & $9 \cdot 34$ & 74.68 & $21 \cdot 34$ & 0.006 & $57 \cdot 34$ & 9.67 & $56 \cdot 34$ & 11.67 & $>0.05$ \\
\hline \multirow[t]{4}{*}{ Catalase $(\mu \mathrm{Kat} / \mathrm{l})$} & $647 \cdot 80$ & 87.68 & $832 \cdot 33$ & 154.53 & $<0.001$ & 663.47 & 66.35 & $656 \cdot 63$ & 54.51 & $>0.05$ \\
\hline & \multicolumn{5}{|c|}{ Between-group changes } & & & & & \\
\hline & \multicolumn{2}{|c|}{ Curcumin } & \multicolumn{2}{|c|}{ Placebo } & & & & & & \\
\hline & Mean & SD & Mean & SD & $P$ & & & & & \\
\hline Substance $P(p g / m l)$ & -1.00 & 0.74 & -0.17 & 0.55 & $<0.001$ & & & & & \\
\hline $\operatorname{SOD}(\mu \mathrm{Kat} / \mathrm{l})$ & $8 \cdot 17$ & $20 \cdot 00$ & -0.83 & $10 \cdot 67$ & 0.015 & & & & & \\
\hline GPx $(\mu \mathrm{Kat} / \mathrm{l})$ & $11 \cdot 17$ & 24.00 & -1.00 & $10 \cdot 84$ & 0.005 & & & & & \\
\hline Catalase $(\mu \mathrm{Kat} / \mathrm{l})$ & $184 \cdot 70$ & 170.53 & $-6 \cdot 83$ & $90 \cdot 52$ & $<0.001$ & & & & & \\
\hline
\end{tabular}

SOD, superoxide dismutase; GPx: glutathione peroxidase. 


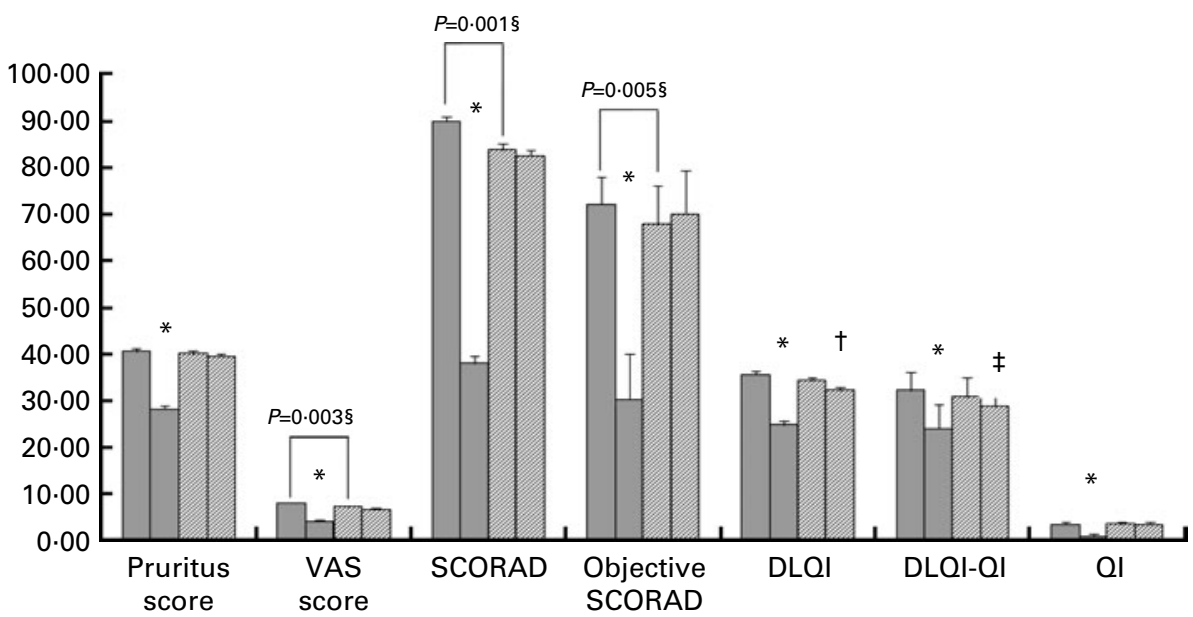

Fig. 2. Pre-trial $v$. post-trial values of the evaluated efficacy measures in the curcumin ( $\square$ ) and placebo (四) groups. VAS, visual analogue scale; SCORAD, scoring atopic dermatitis; DLQI, Dermatology Life Quality Index; $Q_{1}$, first question of the DLQI questionnaire. Values were significantly different: ${ }^{*} P<0.001 ; \dagger P=0.003$; $\ddagger P=0.005$. $§$ Baseline values were significantly different.

$(\beta=-0 \cdot 32,95 \%$ CI $-2 \cdot 78,0 \cdot 06, P=0 \cdot 04)$. No significant predictor was found for changes in VAS score, SCORAD and DLQI. In the placebo group, no significant predictor was found for the evaluated dependent variables.

\section{Discussion}

The results of the present trial imply that curcumin effectively increases the activity of antioxidant enzymes, reduces serum substance $\mathrm{P}$, alleviates pruritic symptoms and increases the QoL in SM-exposed patients who suffer from chronic pruritic skin lesions. To our knowledge, this is the first report on the health effects of curcumin in SM-exposed patients.

There are several reasons for the selection of curcumin as a promising candidate:

(1) Curcumin is a natural compound and the principal constituent of turmeric, an eastern spice that has been consumed by millions of people for over 2000 years, and used in traditional Ayurvedic and Chinese medicines as well. Therefore, curcumin is among the most safest drugs ever known. Curcumin doses of up to $10 \mathrm{~g} / \mathrm{d}$ have been found to be safe and non-toxic in clinical trials $^{(21,30)}$.

(2) Curcumin is endowed with multiple beneficial effects on skin including anti-ageing, rejuvenating and wound healing properties, as well as protection against skin cancer, psoriasis and scleroderma ${ }^{(31,32)}$. Besides, curcumin possesses numerous pleiotropic effects such as cancer chemopreventive, cardioprotective, neuroprotective, anti-diabetic and anti-arthritic activities, which allow its application against a wide array of disorders ${ }^{(22-24)}$.

(3) Curcumin could counterbalance different aspects of SM toxicity such as inflammation, oxidative stress and DNA damage. Inflammation is an important pathomechanism of SM-induced skin toxicity. Several in vitro and in vivo studies have indicated neutrophil infiltration and increased expression of pro-inflammatory cytokines following SM exposure ${ }^{(10,33)}$. On the other hand, curcumin has well-established anti-inflammatory properties and can modulate inflammatory state by interfering with several steps of inflammation pathways that are involved in SM-induced dermatotoxicity including expression and release of pro-inflammatory cytokines (e.g. IL- $1 \alpha$, IL-1 $\beta$, IL-6, TNF- $\alpha$, etc.), transcription factors (NF-кB), cyclooxygenase- 2 , lipoxygenase, mitogen-activated and janus kinases $^{(34-36)}$. An important finding to emerge from the present trial is the significant reduction of substance $P$ following curcumin supplementation. To the best of our knowledge, no study has evaluated the effect of curcumin on serum levels of substance $\mathrm{P}$ nor has been any report on the concentration of this inflammatory and pruritogenic neuropeptide in SM-intoxicated patients. An implication of the present finding is the possibility that substance $\mathrm{P}$ antagonists may be effective drugs against SM-induced

Table 2. Effect of curcumin $v$. placebo on the rate of pruritus improvement

\begin{tabular}{|c|c|c|c|c|c|}
\hline & \multicolumn{2}{|c|}{ Curcumin } & \multicolumn{2}{|c|}{ Placebo } & \multirow[b]{2}{*}{$P$} \\
\hline & $\begin{array}{l}\text { Pre- } \\
\text { trial }\end{array}$ & $\begin{array}{c}\text { Post- } \\
\text { trial }\end{array}$ & $\begin{array}{l}\text { Pre- } \\
\text { trial }\end{array}$ & $\begin{array}{l}\text { Post- } \\
\text { trial }\end{array}$ & \\
\hline \multicolumn{6}{|c|}{ Pruritus score } \\
\hline Mild & 0 & 0 & 0 & 0 & \multirow{3}{*}{$<0.001$} \\
\hline Moderate & 0 & 31 & 2 & 2 & \\
\hline Severe & 40 & 9 & 38 & 38 & \\
\hline \multicolumn{6}{|l|}{ VAS score } \\
\hline Mild & 0 & 16 & 0 & 1 & \multirow{3}{*}{$<0.001$} \\
\hline Moderate & 2 & 20 & 12 & 15 & \\
\hline Severe & 38 & 4 & 28 & 24 & \\
\hline \multicolumn{6}{|c|}{ Overall SCORAD } \\
\hline Mild & 0 & 0 & 0 & 0 & \multirow{3}{*}{$<0.001$} \\
\hline Moderate & 0 & 37 & 0 & 0 & \\
\hline Severe & 40 & 3 & 40 & 40 & \\
\hline \multicolumn{6}{|c|}{ Objective SCORAD } \\
\hline Mild & 0 & 0 & 0 & 0 & \multirow{3}{*}{$<0.001$} \\
\hline Moderate & 0 & 36 & 0 & 0 & \\
\hline Severe & 40 & 4 & 40 & 40 & \\
\hline
\end{tabular}

VAS, visual analogue scale; SCORAD, scoring atopic dermatitis. 
pruritus and perhaps other inflammatory complications of chemically injured veterans.

To date, data from several studies have identified oxidative stress as an important factor involved in SM-induced skin toxicity $^{(10,25,33)}$. Development of oxidative stress could be attributed to the propensity of SM to react with sulfhydryls and intracellular reductases, thereby depleting cellular antioxidants ${ }^{(33,37)}$. On the other hand, recruited inflammatory cells generate reactive oxygen species that further contribute to oxidative stress. In a previous investigation, plasma, erythrocyte and bronchoalveolar fluid activities of SOD, GPx and catalase have been found to be significantly lower in SM-injured patients compared with patients with chronic respiratory disease without a history of SM exposure ${ }^{(38)}$. The present findings indicate the enhancement of these endogenous antioxidants by curcumin therapy. This may also confirm the importance of antioxidant therapy in the management of SM-induced complications. Both in vitro (on cultured human keratinocytes) and in vivo evidence suggest that pre-treatment with antioxidants attenuates SM-induced dermatotoxicity $^{(25,39,40)}$. However, in spite of well-documented antioxidant activities of curcumin, this phytochemical has not been investigated in relation to SM-related toxicity. Curcumin could counteract oxidative stress via several mechanisms including free-radical-scavenging activity, enhancement of intracellular glutathione concentrations and inhibition of lipid peroxidation. In addition, by triggering the nuclear factor-erythroid 2-related factor 2/antioxidant response element signalling pathway, curcumin could activate antioxidant enzymes and members of the vitagene family such as haem oxygenase, Hsp70 (heat shock protein 70) and thioredoxin reductase ${ }^{(41)}$. On the other hand, given the close link that exists between inflammation and oxidative stress, curcumin's anti-inflammatory effects - mainly promoted via the inhibition of NF-кB, cyclo-oxygenase-2, lipoxygenase and inducible NO synthase

- could help to attenuate oxidative stress and stop the vicious inflammatory/oxidative cycle of SM toxicity ${ }^{(42,43)}$.

Finally, curcumin has been reported to possess antimutagenic and anti-genotoxic effects ${ }^{(44,45)}$. Therefore, it has the potential to revert DNA cross-linking, chromosome aberrations and abnormalities that are sequelae of SM toxicity.

In the present study, significant improvements in QoL were observed in both curcumin and placebo groups, though the extent of the reduction in DLQI and its subcategories (DLQIQ1 and Q1 scores) was significantly greater in the curcumin $v$. placebo group. A so-called 'placebo effect' (psychological or psychophysiological response which causes improvement of symptoms) might be responsible for the observed improvement of QoL in the placebo group ${ }^{(46)}$. In addition, piperine, which was present in the placebo capsules, is a bioactive alkaloid that may exert additional beneficial effects such as anti-inflammatory and anti-nociceptive effects ${ }^{(47,48)}$. Therefore, these beneficial effects could also be responsible for QoL improvement in the placebo group.

Among the previously performed trials, the rate of recovery based on the pruritus score (but not the overall or objective SCORAD) could be extracted for $0.1 \%$ betamethasone cream, pimecrolimus, capsaicin and phenol/menthol. Interestingly, the rate of pruritus improvement by curcumin (77.5\%) is higher compared with all aforementioned medications, namely $0 \cdot 1 \%$ betamethasone cream $(62.5 \%$, 6-week trial), pimecrolimus $(68.8 \%, 6$-week trial), capsaicin $(35.5 \%$, 6-week trial) and phenol/menthol $\left(60 \%\right.$, 6-week trial) ${ }^{(16-18)}$ This is especially important when considering the huge pleiotropic benefits from curcumin. In the present trial, the passage of severity into the mild category did not occur for the pruritus, overall SCORAD and objective SCORAD scores. However, in previous trials with other medications (with a longer duration of 6 weeks), the passage of pruritus score severity into the mild category has been reported but what may be more important is moving into a less severe category. Regression of severity also depends on the baseline severity of pruritus. For instance, all participants in the present study had severe pruritus at baseline. Taken together, comparison of the improvement rates in the present trial with those of previously published studies on other medications does not negate the need for a direct comparative trial between curcumin and other routinely administered medications. As this was the first trial investigating curcumin's efficacy against SM-induced pruritus, the findings may generate hypotheses for future trials to compare curcumin $v$. other routinely administered drugs for SM-induced pruritus.

As another implication, the present study confirms previous findings and contributes additional evidence that suggests a favourable therapeutic response following co-administration of curcumin with piperine ${ }^{(49)}$. Piperine is a bioactive alkaloid present in the fruits of $P$. nigrum $\mathrm{L}$. (black pepper) or $P$. longum L. (long pepper). This phytochemical is regarded as a natural and powerful bioavailability enhancer capable of increasing the absorption of a wide variety of nutrients ${ }^{(26)}$. The major obstacle for the therapeutic effectiveness of oral curcumin supplementation is its low bioavailability, which is secondary to the relatively poor systemic absorption of this compound from the gastrointestinal tract as well as rapid metabolism $^{(50,51)}$. Therefore, co-administration of curcumin and piperine has been proposed as an effective strategy for the enhancement of curcumin bioavailability. Several proofof-concept studies have indicated that this combination can boost the bioavailability of curcumin as high as $2000 \%{ }^{(26)}$.

In summary, the most obvious finding to emerge from the present study is that supplementation of curcumin is associated with marked alleviation of chronic pruritus in SM-intoxicated patients. In light of its favourable impacts on pruritus and other skin complications, curcumin could also increase the patients' QoL. The second major finding was the elevation of serum antioxidant enzymes and a marked reduction of substance P by curcumin therapy, which could be regarded as an important mechanism for the observed anti-pruritic effects. Taken together, the present findings clearly support the relevance of curcumin as an effective, safe, widely available and inexpensive treatment for the management of SM-induced chronic pruritic skin lesions. However, further research needs to be undertaken in order to unravel the impact of other curcumin doses and treatment durations as well as mechanisms behind the beneficial effects of curcumin against SM 
dermatotoxicity. To address this latter issue, estimation of curcumin's influence on other measures of oxidative stress as well as on other aspects of SM toxicity is recommended.

\section{Acknowledgements}

This study was financially supported by the Baqiyatallah University of Medical Sciences (Tehran, Iran). The authors acknowledge with grateful appreciation Sami Labs Limited (Bangalore, India) for its cooperation in providing curcumin and placebo capsules, as well as Mehrdad Iranshahi for his kind assistance in conducting the study. Y. P. and A. S. discussed the core ideas of the protocol design and/or handled the execution of the study. M. A., S. M. D., S. L. H. and M. K. were responsible for patient recruitment, visit and/or sample collection. Y. P. and A. S. were involved in the data analysis and interpretation, literature review and drafting of the manuscript. There are no conflicts of interest.

\section{References}

1. Wormser U (1991) Toxicology of mustard gas. Trends Pharmacol Sci 12, 164-167.

2. Kehe K \& Szinicz L (2005) Medical aspects of sulphur mustard poisoning. Toxicology 214, 198-209.

3. Khateri S, Ghanei M, Keshavarz S, et al. (2003) Incidence of lung, eye, and skin lesions as late complications in 34,000 Iranians with wartime exposure to mustard agent. J Occup Environ Med 45, 1136-1143.

4. United Nations Security Council (1986) Reports of the Specialists Appointed by the Secretary General to Investigate Allegations by the Islamic Republic of Iran Concerning the Use of Chemical Weapons. Document S/16433. New York, NY: Security Council of the United Nations.

5. Balali-Mood M \& Hefazi M (2005) The clinical toxicology of sulfur mustard. Arch Iranian Med 8, 162-179.

6. Shohrati M, Peyman M, Peyman A, et al. (2007) Cutaneous and ocular late complications of sulfur mustard in Iranian veterans. Cutan Ocul Toxicol 26, 73-81.

7. Panahi Y, Davoudi SM, Sadr SB, et al. (2008) Impact of pruritus on quality of life in sulfur mustard-exposed Iranian veterans. Int J Dermatol 47, 557-561.

8. Weisshaar E \& Diepgen TL (2005) Pruritus and quality of life. J Invest Dermatol 125, 855-855.

9. Weisshaar E, Apfelbracher C, Jager G, et al. (2006) Pruritus as a leading symptom: clinical characteristics and quality of life in German and Ugandan patients. Br J Dermatol 155, 957-964.

10. Ghabili K, Agutter PS, Ghanei M, et al. (2011) Sulfur mustard toxicity: history, chemistry, pharmacokinetics, and pharmacodynamics. Crit Rev Toxicol 41, 384-403.

11. Naghii MR (2002) Sulfur mustard intoxication, oxidative stress, and antioxidants. Mil Med 167, 573-575.

12. Thomsen JS, Sonne M, Benfeldt E, et al. (2002) Experimental itch in sodium lauryl sulphate-inflamed and normal skin in humans: a randomized, double-blind, placebo-controlled study of histamine and other inducers of itch. Br J Dermatol 146, 792-800

13. Hägermark O, Hökfelt T \& Pernow B (1978) Flare and itch induced by substance P in human skin. I Invest Dermatol 71, 233-235.
14. Lotz M, Vaughan JH \& Carson DA (1988) Effect of neuropeptides on production of inflammatory cytokines by human monocytes. Science 241, 1218-1221.

15. Lieb K, Fiebich BL, Berger M, et al. (1997) The neuropeptide substance $\mathrm{P}$ activates transcription factor NF-kappa B and kappa B-dependent gene expression in human astrocytoma cells. J Immunol 159, 4952-4958.

16. Panahi Y, Davoodi SM, Khalili H, et al. (2007) Phenol and menthol in the treatment of chronic skin lesions following mustard gas exposure. Singapore Med J 48, 392-395.

17. Panahi Y, Moharamzad Y, Beiraghdar F, et al. (2009) Comparison of clinical efficacy of topical pimecrolimus with betamethasone in chronic skin lesions due to sulfur mustard exposure: a randomized, investigator-blind study. Basic Clin Pharmacol Toxicol 104, 171-175.

18. Panahi Y, Davoudi SM, Moharamzad Y, et al. (2008) Comparison of topical capsaicin and betamethasone in the treatment of chronic skin lesions due to sulfur mustard exposure. Cutan Ocul Toxicol 27, 203-211.

19. Shohrati M, Davoudi M, Almasi M, et al. (2007) Comparative study of Unna's Boot and betamethasone cream in the treatment of sulfur mustard-related pruritus. Cutan Ocul Toxicol 26, 303-309.

20. Shohrati M, Davoudi SM, Keshavarz S, et al. (2007) Cetirizine, doxepine, and hydroxyzine in the treatment of pruritus due to sulfur mustard: a randomized clinical trial. Cutan Ocul Toxicol 26, 249-255.

21. Henqque UR, Ruzicka T, Schwartz RA, et al. (2006) Adverse effects of topical glucocorticosteroids. J Am Acad Dermatol 54, 1-15.

22. Pari L, Tewas D \& Eckel J (2008) Role of curcumin in health and disease. Arch Physiol Biochem 114, 127-149.

23. Goel A, Kunnumakkara AB \& Aggarwal BB (2008) Curcumin as "Curecumin": from kitchen to clinic. Biochem Pharmacol 75, 787-809.

24. Epstein J, Sanderson IR \& Macdonald TT (2010) Curcumin as a therapeutic agent: the evidence from in vitro, animal and human studies. Br J Nutr 103, 1545-1557.

25. Paromov V, Suntres Z, Smith M, et al. (2007) Sulfur mustard toxicity following dermal exposure: role of oxidative stress, and antioxidant therapy. J Burns Wounds 7, e7.

26. Shoba G, Joy D, Joseph T, et al. (1998) Influence of piperine on the pharmacokinetics of curcumin in animals and human volunteers. Planta Med 64, 353-356.

27. Rickham PP (1964) Human experimentation. code of ethics of the world medical association. Declaration of Helsinki. Br Med J 2, 177.

28. Balaskas EV, Bamihas GI, Karamouzis M, et al. (1998) Histamine and serotonin in uremic pruritus: effect of ondansetron in CAPD-pruritic patients. Nephron 78, 395-402.

29. European Task Force on Atopic Dermatitis (1993) Severity scoring of atopic dermatitis: the SCORAD Index. Consensus Report of the European Task Force on Atopic Dermatitis. Dermatology 186, 23-31.

30. Aggarwal BB, Kumar A \& Bharti AC (2003) Anticancer potential of curcumin: preclinical and clinical studies. Anticancer Res 23, 363-398.

31. Chainani-Wu N (2003) Safety and anti-inflammatory activity of curcumin: a component of tumeric (Curcuma longa). J Altern Complement Med 9, 161-168.

32. Thangapazham RL, Sharma A \& Maheshwari RK (2007) Beneficial role of curcumin in skin diseases. Adv Exp Med Biol 595, 343-357.

33. Sikora E, Scapagnini G \& Barbagallo M (2010) Curcumin, inflammation, ageing and age-related diseases. Immun Ageing 7, 1. 
34. Shakarjian MP, Heck DE, Gray JP, et al. (2010) Mechanisms mediating the vesicant actions of sulfur mustard after cutaneous exposure. Toxicol Sci 114, 5-19.

35. Jin CY, Lee JD, Park C, et al. (2007) Curcumin attenuates the release of pro-inflammatory cytokines in lipopolysaccharide-stimulated BV2 microglia. Acta Pharmacol Sin 28, $1645-1651$.

36. Abe Y, Hashimoto S \& Horie T (1999) Curcumin inhibition of inflammatory cytokine production by human peripheral blood monocytes and alveolar macrophages. Pharmacol Res 39, 41-47.

37. Jurenka JS (2009) Anti-inflammatory properties of curcumin, a major constituent of Curcuma longa: a review of preclinical and clinical research. Altern Med Rev 14, 141-153.

38. Brimfield A, Mancebo A, Mason R, et al. (2009) Free radical production from the interaction of 2-chloroethyl vesicants (mustard gas) with pyridine nucleotide-driven flavoprotein electron transport systems. Toxicol Appl Pharmacol 234, $128-134$.

39. Jafari M \& Ghanei M (2010) Evaluation of plasma, erythrocytes, and bronchoalveolar lavage fluid antioxidant defense system in sulfur mustard-injured patients. Clin Toxicol (Phila) 48, 184-192.

40. Eldad A, Ben Meir P, Breiterman S, et al. (1998) Superoxide dismutase (SOD) for mustard gas burns. Burns 24, 114-119.

41. Gross CL, Nealley EW, Nipwoda MT, et al. (2006) Pretreatment of human epidermal keratinocytes with DL-sulforaphane protects against sulfur mustard cytotoxicity. Cutan Ocul Toxicol 25, 155-163.

42. Calabrese V, Bates TE, Mancuso C, et al. (2008) Curcumin and the cellular stress response in free radical-related diseases. Mol Nutr Food Res 52, 1062-1073.
43. Menon VP \& Sudheer AR (2007) Antioxidant and antiinflammatory properties of curcumin. Adv Exp Med Biol 595, 105-125.

44. Bengmark S (2006) Curcumin, an atoxic antioxidant and natural NFкB, cyclooxygenase-2, lipooxygenase, and inducible nitric oxide synthase inhibitor: a shield against acute and chronic diseases. JPEN J Parenter Enteral Nutr 30, 45-51.

45. Ragunathan I \& Panneerselvam N (2007) Antimutagenic potential of curcumin on chromosomal aberrations in Allium cepa. J Zhejiang Univ Sci B 8, 470-475.

46. de Craen AJ, Kaptchuk TJ, Tijssen JG, et al. (1999) Placebos and placebo effects in medicine: historical overview. $J R$ Soc Med 92, 511-515.

47. Bang JS, Oh da H, Choi HM, et al. (2009) Anti-inflammatory and antiarthritic effects of piperine in human interleukin 1beta-stimulated fibroblast-like synoviocytes and in rat arthritis models. Arthritis Res Ther 11, R49.

48. Srinivasan K (2007) Black pepper and its pungent principlepiperine: a review of diverse physiological effects. Crit Rev Food Sci Nutr 47, 735-748.

49. Durgaprasad S, Pai CG, Vasanthkumar JFA, et al. (2005) A pilot study of the antioxidant effect of curcumin in tropical pancreatitis. Indian J Med Res 122, 315-318.

50. Anand P, Kunnumakkara AB, Newman RA, et al. (2007) Bioavailability of curcumin: problems and promises. $\mathrm{Mol}$ Pharm 4, 807-818.

51. Sharma RA, Steward WP \& Gescher AJ (2007) Pharmacokinetics and pharmacodynamics of curcumin. Adv Exp Med Biol 595, 453-470. 